\title{
Apparatus to Detect Stable Fractional Charges on Matter
}

\author{
Roland Vanderspek \\ Lawrence. Berkeley Laburatury \\ University of California \\ Berkeley, California 94720
}

\section{Introduction}

In the early years of this century, Millikan demonstrated that the electric charges on tiny droplets of oil were quantized. With this discovery he confirmed that matter was made up of particles charged with integral-multiples of a basic charge unit $e^{\prime}=4.80 \times 10^{-11)}$ ess. In the nid60's Gell-Mann and Zweig proposed a theory of hadron structure based on three types of quarks, objects which are most easily interpreted as particles with charges of $\pm \frac{1}{3} e^{\prime}$ or $\pm \frac{2}{3}$ '. Later experiments bore out the theory by showing thal hadrons appear to be composed of point-like fractionally-charged particles. One question raised by these results is that of the existence of an isolated quark in nature. There have been many searches for fractionallycharged particles produced in high-energy accelerator reactions and in even higher-energy cosmic-ray events, but they have yielded no positive results. - If quarks exist at all in isolation. there is reason to belicve that they will be found in some concentration in the Earth's crust. The quarks would most likely be the result of some super-high-energy cosmic-ray event or the relics of some early stage of the formation of the uni, serse. Many searches for isolated quarks on slable matter have been undertaken, again with no positive results.? The total anount of matter examined direclly in the searches to date is a few ni:ligrams. An apparalus is described here which will analyze malter for stable fractional charges in quantities ranging around one gram. A rate of mass-analysis of $10^{-1} \mathrm{~g} / \mathrm{s}^{\prime} \mathrm{c}$ and an ultimate sensitivity of $10^{-24}$ per nucleon are expected.

\section{Theory of Apparatus}

The apparatus analyzes the charges of a series of droplets by measuring their deflectirn in falling through a horizontal static electric field. The force on a droplet is

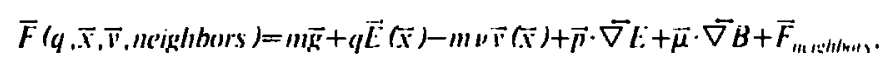

n'here' $q=$ charge' on drople't

$\vec{v}=\mathrm{ve} /$ ocity of dioplet 
$m=$ inass of droplet

$\vec{g}=$ gravitational acceleration

$\vec{\nabla} E=$ electric field gradient

$\vec{\nabla} B=$ magnetic field grudicut

$\nu=$ drag coefficient

$\vec{p}=e$ eccric dipole moment of droplet

$\vec{\mu}=$ magnetic dipole moment of droplet.

The term $\vec{F}_{\text {newhiturs }}$ is the total force due to the electrical and aerodynamic interactions with nearby droplets in the apparatus. These electrical interactions are between the respective charges and dipole moments of neighboring droplets. The dominant term in $\bar{F}_{\text {urrothur }}$ is that of the dipole-dipole interaction between adjacent droplets. The magnitude of these forces decreases with distance, so minimization of their effects requires the maintenance of a minimum separation between droplets. This allows the simplification of the dependence of the force on a droplet to $\vec{F}(q, \vec{v}, \vec{x})$. The net deflection of a droplet by this force is

$$
\delta x=\frac{q V_{0} L^{2}}{m \nu^{2} b},
$$

where $V_{0}=$ potential on the deflecting electrodes

$L=$ length of deflecting electrodes

$b=$ mean separation betueen deflecting electrodes

$\nu=$ characteristic velocity of droplet.

In the ideal case, where all parameters besides droplet charge are repeatable, the deflection of all droplets of a given charge due to this force is the same for each droplet, and the precision of resolution of the charge is limited only by the resolution in the measurement of the deflection. In actuality, the deflection of a given charge varies with the parameters. High charge resolution requires high consistency in these parameters; i.e. the variations $\delta \mathrm{m} / \mathrm{m}$ and $\delta \mathrm{v} / \mathrm{v}$ among all droplets must be kept small. The existence of a divergent spray in the stream of droplets introduces an essentially random component of velocity yerpendicular to the flow to the droplets. All perpendicuiar velocities musı be kepl to an absolute minimum, since a small velocity, multiplied by a time-of-flight of the order of a second, can result in a noticeable variance in the deflection of a droplet. It is also important that none of the forces or parameters change with 
time. Such time-variations would change the deflection of droplets of a given charge from one droplet to the next and thereby lower the resolution of analysis.

The series of droplets is formed from a stream of liquid emitted by the apparatus. The production of droplet jets from streams of liquid depends on the tendency of a liquid to minimize its surface energy by minimizing its surface area. The lowest surface energy configuration of a given volume of liquid is a single sphere. A cylindrical stream of radius a can therefore lower its surface energy by breaking up into a series of identical spheres of separation $d$ for certain values of $d$. The ratio of surface area of the stream to that of the spheres is proportional to $(d / a)^{1 / 3}$. Exact analysis reveals that the stream is energetically favored to break up into droplets with $d>4.5 a$. The existence of such lower surface energy configuritions makes the stream inherently unstable. Thus, the proper perturbations to the surface of the stream will grow to achieve these configurations.

Because of the instability of the stream, a capillary wave applied to its surface will grow in ampitude until droplets are formed (sec Figure 1). For the purposes of calculatinn, we assume the capillary wave to be a small sinusoidal perturbation whose amplitude grows as $e^{\prime \prime \prime}$. In the growth process, the surface potential energy of the stream decreases as the surface area decreases, while, at the same time, certain parts of the stream experience an increase in kinelic energy in contracting into droplets. Conservation of energy leads to the equation

$$
\nu^{2}=\text { constant } \times\left(\frac{\pi k^{2}}{V / L}-k^{4}\right)
$$

where $k$ is the wave number of the perturbation and $V / L$ is the volume per unit length of the stream. This function has a sharp maximum, $\nu_{\text {max }}$, at a certain wavelength perturbation, $k_{\text {t.mmumt }}$ (see Figure 2). This wavelength corresponds to a capillary-wave frequency of $\omega_{\text {rymumtrt }}=v k_{\text {thttmmat }}$, where $v$ is the velocity of the stream. The growlh rates of higher harmonics of this frequency,

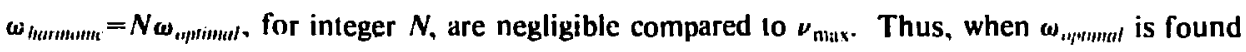
in combination with higher harmonics in a complex perturbation it is the dominant frequency, and the effects of the higher perturbation frequencies are negligible. Sub-harnonic perturbations, on the other hand, could introduce cyclic variations in the droplet rarameters. The droplet radius $r$, the droplet separalion $d$ and $k_{\text {iystrmut }}$ are all functions of the stream radius $a$. In the simple approximation presented above, $k_{\text {ipmumat }}=0.707 / a$. $\wedge$ perturbation of this wave number produces droplets of radius $r=1.88 a$ and separation $d=4.72 r$. In aclual practice, $k_{\text {i,yumal }} \approx 0.70 / a$, producing droplets of $r \approx 1.89 a$ and $d \approx 4.77 r{ }^{3}$

Non-linear effects associated with certain combinations in wavelength and anplitude capillary-wave perturbations are conducive to the formation of smaller, shtellite droplets between adjacent primary droplets (see Figure $1 d$ ). It is beyond the scope of this paper to go 
into the details of this phenomenon, as the theory of the formation of satellites has not yet been developed. Figure 3 is a plot of data collected by researchers at $I B M$ and indicates the frequency-amplitude window in which satellites are created. ${ }^{4}$ At amplitudes above the window, the satellites will forward-merge within a few wavelengths; at those below the window, the satellites backward-merge in the same range. The diameter of a satellite droplet is generally about onc tenth the diameter of a primary droplet.

A chars. can be induced on a droplet by applying a potential to an electrode surrounding the stream at the point of separation. The electrically conductive liquid in the stream is held near zero potentia: thus, the potential on the electrode draws charges into the tip of the stream. The magnitude of the mean charge induced on the droplet depends on the capacitance between the droplet and the electrode and the capacitances between the droplet and previously released droplets Thermal fluctuations produce a distribution of charges around this mean charge. The rms deviation of the distribution of the charges on a capacitor $C$ in equilibrium with a temperature bath $T$ is equal to $\sqrt{2 C k T}$. For an isolated spherical droplet, the capacitance is jusl the droplet radius.

\section{Description of Method}

The apparatus, which is diagrammed in Figure 4, can operate in two modes. Both modes produce droplets at rates corresponding to maxinum growth of capillary-wave perlurbations. The first mode produces a series of small, identical droplets of which only a small percentage is retained for analysis. These droplets are given a mean charge of zero; the thermally-induced deviations about this mean are analyzed. The remaining droplets are charged highly and deflected from between the deflecting electrodes in order to increase the separation between analyzed droplets. A small slot in each of the deflecting electrodes facilitales the disposal of these and other highly-charged droplets.

The second mode operates in the satellite-creation window (see Figure 3), and produces satellites between primary droplets comparable in size to the primary droplets of mode 1 . A larger orifice produces larger, more-widely separated primary droplets than in mode 1. All droplets are given zero mean charge, and the satellite droplets are charge-analyzed as above. The primary droplets are removed from analysis by striking and wetling a small silver-plated sphere before passing between the deflecting electrodes. This sphere makes grazing contact with the primary droplets and no contact with the satellites. The prinary droplets adhere to the wetled surface and are carried along the sphere, being released from the surface after experiencing deflections of about 30 degrees. The liquid of the droplet collects in a doughnut-shaped well surrounding the stream of smaller droplets. The well also serves to collect mercury dripping from the silvered sphere. The liquid in this well flows through a drain into a second well, from 
which it flows out of the apparatus through a section of stainless steel pipe. The primary droplets can also be allowed to travel with the satellites, falling essentially undeflected through the apparatus. In both modes analyzed droplets maintain at least the separation calculated to reduce adequately the effects of electrical and aerodynamic interactions. This separation is approximately $650 \mu \mathrm{m}$ for $10 \mu \mathrm{m}$ drops.'

The capillary-wave perturbation to the surface of the stream is provided by two piezoelecIric crystals. An oscillating potential across the crystals causes them to vibrate parallel to their axis as in Figure 5. Th.e longitudinal vibrations of the crystals and the throat of the droplet generator coupled with the induced vibrations in the stream and orifice introduce harmonic perturbations of higher frequencies to the stream. These harmonics and the variations of the driving signal from a pure sinusoid have little effect on the formation of droplets. The dynamics of capillary-wave growth, as discussed above, emphasizes only one of the set of harmonics making up a complex wave-form, and thus renders higher harmonics harmless.

\section{Apparatus}

The droplet generator is made of electron-beam welded $A I S / 412$ stainless steel. II is diagrammed in Figures 4 and 6 . In mode 1, the droplet generator produces on the order of $10^{5}$ droplets of mercury per second, with $\rightarrow 10 \mu m$-diameter orifice producing $20 \mu m$-diameter droplets. The orifice from which the stream is emitted is a small hole drilled into a thin stainless steel sheet. In mode 2 , the droplet generator produces $\approx 10^{4} 200 \mu \mathrm{m}$ droplcts $/ \mathrm{scc}$ from a $100 \mu \mathrm{m}$ orifice. In both cases the initial velocity of the droplets is near $500 \mathrm{~cm} / \mathrm{sec}$. The droplet gen-

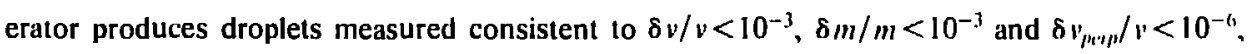
where $v_{p r q}$ is the component of droplet velocity perpendicular to the flow of droplets. The stream of droplets may be observed before deflection with a microscope situated above the deflecting electrodes.

The charging electrode is a cylinder of high-transparency etched nickel mesh concentric to the stream of liquid at the point of separation of the droplets. Application of a $3 \mathrm{mV}$ potential to the charging electrode will induce a mean charge of $10 e$ on a droplet. The charge induced on a droplet is highly sensitive to changes in the potential applied to the charging electrode; hence, the applied potential must be very accurate in order to be able consistently to induce the same mean charge on each of $:$ series of droplets. In mode 1 , approximately 29 of every 30 droplets are charged to about $300 e$ by the application of a $0.1 V$ potential to the charging electrode. These droplets are deflected from between the deflecting electrodes within a distance of $3 \mathrm{c} / \mathrm{m}$. This stream of droplets is inherently unstable because of the mutual repulsion of the charges on the droplets. The application of higher charges to these droplets would increase this instability and thereby increase the magnitude of their random effects on the trajectories of the analyzed 
droplets. The induction of zero mean charge on the remaining droplet requires the application of a potential to the charging electrode to cancel the contact potentials on the stream. The magnitude of this potential is roughly $0.5 \mathrm{~V}$. The $r m s$ deviation from zero charge due to thermal effects is calculated to be approximately $\pm 18 \mathrm{c}$ on $10 \mu \mathrm{m}$ droplets. This calculation has been independently veritied by Roger Bland of San Francisco State University, who measured an rm.s deviation of $\pm 12 c^{\prime}$ on $5 \mu m$ droplets.

The initial signal to the charging electrode is the same $A C$ signal ditiving the piezoelectric crystals. This signal is changed into a series of standard $-0.85 \mathrm{~V} N \mathrm{M}$ pulses of the same frequency by a discriminator. The pre-scaler suppresses all but one of every $N$ of these pulses, where $N$ is selectable from I to 15; the pulse not suppressed appears at the output as a square $N I M$ pulse of one cycle width. Several pre-scialings in series allow the number of suppressed cycles to be increased. The pulse generator can change the width and phase with respect to the driving oscillation of this pulse. The charging electrode driver changes the amplitude and bias of the pulse; it can apply an arbitrary polential to the charging electrode with a measured accuracy of $10 \mu \mathrm{V}$. The output of the discriminator is also fed to a logical $A N D$ circuit and a gate in series. The output of the gate, which is the second input of the $A N D$, is normally in the high logical state. The input of a pulse changes the output of the gate from the high state to the low state for a specifiable time and thereby forces the output of the $A N D$ into the low state for the same period. After this time period the gate relurns to the high state, whereupon the first pulse passing from the $A N D$ sends the output of the gate back into the low statc. The frequency of the series of pulses from the discriminator can be lowered in this manner. The lowered frequency of pulses coming from the $A N D$ is used to drive the strobe with which the droplets are observed.

The reservoir of the droplet generator is pressurized to approximately 30 psi with Argon gas. The regulation of the pressure is important because the velocity of the strean is dependent on the pressure. The pressure is regulated by a constant-bleed regulator in series with a standard regulator and is steady to about $0.1 \%$ height of the column of mercury in the reservoir is gradual and steady and less than $101 / 1 / \mathrm{hrm}$. The time-scale of analysis is timiled by the loss of resolution due to the change in velocity associated with this variation in pressure. Pressure valves on the electrode housing and the nercury trap guard against catastrophic increases in pressure in the apparatus. Both valves trip at a pressure 3 psi over atmospheric.

The deflecting electrodes are 36 -inch sections of inch-wide, sixteenth-inch-thick chromeplated ground steel. The geometry of the electrodes is detailed in Figure 7. The electric field used in actual operation is produced by a $3 \mathrm{kV}$ potential across the electrodes. The resulting difference between the deflections of adjaceni integer charges is predicted to be $0.7 \mathrm{~mm}$. All 
areas of low radius of curvature must be removed from the electrodes before operation in order to minimize field-ion emission associated with the intensified electric field at these points. The corners and edges of the steel were rounded prior to plating, and the chrome itself is smooih to $60-L$ specification $(\approx 80 " A$ peak-to-peak variation). The ion-bombardment associated with the field-emission erodes any points on the negative deflecting electrode to an extent dependent on the magnitude of the applied electric field. In this manner, the deflecting electrodes are electro-polished before use by a $10 k v$ potential of each polarity, applied across the electrodes until the ion-current between them disappears.

The molecular sieve, Zeolite, used in the evacuation of the apparatus will absorb most vapors when cooled to $L N_{2}$ temperatures. The system is mechanically pumped down to a pressure of $100 \mu H_{k}$ before exposing the Zeolite. This reduces the concentration of the few elements Zeolite is unable to absorb and so allows realization of the required $2 \mu H_{k}$ pressure. The vapor from the housing passes through the mercury trap, which is filled with copper wool. The copper amalgamates with any mercury vapor present and so removes the vapor from the system. The remaining vapor passes into a $L N_{2}$ cold trap, where it is exposed to the Zeolite. The Zeolite pump contributes no vibrational noise to the system.

The photo-ejection of charges from the droplets might lead to a build-up of static electric charge on the dielectric inside surface of the housing pipe. This charge would have unpredictable effects on the trajectories of the charged droplets. A thin, electrically grounded layer of conductive graphite coating the inside of the housing pipe prevents such a buildup of chargc. The observation windows in the apparatus are screened off with stainless steel mesh to complete the electrostatic shielding and at the same time preserve the transparency of the windows. The resistance of the graphite layer is on the order of $100 \mathrm{k} \Omega / \mathrm{fl}$. Precautions were taken to minimize the photo-ejection of charge by making the apparatus opaque to ultrat-violet light. The observalion windows are made of $U V$-absorbing acrylic, and any $U V$-leaks into the apparatus are hlacked over. The analysis of the droplets takes place in a light-tight box fixed at the bottom of the housing pipe.

The initial analysis of droplet charge will be made with a simple still camera. The film is exposed to light reflected from the droplets for a period of the order of a minute. The light illuminating the droplets is from a strobe synchronized to the rate of passage of droplets. The intensity of the image of a stream of deflected droplets depends on the number of droplets in the stream. Brighter streams are most likely to be those of integrally-charged droplets; anyt fainter stream between integer streams might correspond to a fractional charge.

The data are taken with a 1728-bucket optically-sensitive charge-coupled device (C $C D$ ). Light from a gas laser is scattered from the droplets onto the array of buckets. Each bucket 
develops a charge which is proportional to the amount of light incident during the period of data accumulation. This period corresponds to the passage of a few droplets through the beam. Each bucket is in two sections in order to be able to collect new data while old data is being analyzed. A "start-of-scan" signal from the C $C D$ relocates the charges to the lower half of the array, and the data analysis is initiated. The charges in the array of buckels are all physically shifted, in sleps of one bucket, to the end of the array, where the contents of each buckel can be individually amplified and fed into a discriminator. The C $C D$ is set 10 initiate a new round of data analysis as soon as the contents of all the buckets from the previous scan have becn read. The discriminator outputs a slandard high $T T L$ pulse upon reception of a signal of amplilude greatei than $\approx 0.1 \mathrm{~V}$. The pulse height delivered from the passage of onc droplet is $\approx 0.3 \mathrm{~V}$. The discriminator output is fed 10 a multichannel analyzer, which sorts incoming puises into its 1024 storage bins according to time of arrival. The "start-of-scan" signal from the $C C D$ defines the zero of time for the multichannel analyzer. The multichannel analyzer can accept and store only one pulse per definable period of time. The smallest period definable is $10 \mu \mathrm{sec}$, corresponding to a maximum rate of pulse analysis of $100 \mathrm{kHz}$. However, the $(C D$ oulputs the data from the array at rates helween $156 \mathrm{kHz}$ and $10 \mathrm{MHz}$. Running the multichannel analyzer at its maxinum rate and the $C C D$ at its lowest rate produces acceptable results. The low rate of analysis of $C C D$-bucket contents allows noise of the order of $0.01 V$ to build up in the buckets.

\section{Conclusion}

No data had been taken up to the time of publication. From the tests made of the apparatus we feel that the predicted sensitivity of $10^{-24} /$ moken can be atlained. The apparatus is designed to produce a separation of $\approx 700 \mu \mathrm{m}$ between adjacent integer charges. Earlier calculations of the magnitude of random effects on the trajectories of the droplets show that the maximum variance of a charged droplet from its unperturbed trajectory is $8 \mu \mathrm{m}$, thus, an accuracy of better than $0.02 e$ can be achieved with the apparalus as described above.'

\section{Acknowledgements}

I would like to thank Greg Hirsch and Ray Hagstrom of LBL for their help in the preparation of this document.

Prepared for the U.S. Department of Energy under Contract W-7405-ENG-48 


\section{References}

1. G. Hirsch, R. Hagstrom, C. Hendricks, $L B L$ Report No. $L B L-9350,1979$ (unpublished).

2. L. W. Jones, "A Review of Quark Search Experiments", Reviews of Modern Physics, 49, 717 (1977).

3. H. C. Lee, "Drop Formation in a Liquid Jet", IBM Journal of Researd and De'selopmen'mt. 18, 364 (1974).

4. W.T. Pimbley and H.C. Lee. "Satellite Droplet Formation in a Liquid Jet", IBM .Iournal of Research and Development, 21, 2 I (1977).

5. R. Bland (personal communication). 


\section{Figure Captions}

Fig. 1. Capillary-wave growth. A capillary-wave perturbation applied to a cylindrical stream of liquid $(a)$ will grow $(b)$ until droplets are formed $(c)$. The radius of the stream is given by $r(z)=a+p(t) \sin (k z)$, where $a$ is the radius of the liquid strcam, $\rho(t)$ is the time-varying amplitude of the capillary-wave perturbation, and = is mcasured along the axis of the stream. Certain combinations of amplitude and frequency of capillary-wave perturbation induce the production and retention of satellite droplets between primary droplets $(d)$. The satellites are formed from the isthmus of liquid between primary droplets.

Fig. 2. The dependence of the rate of growth " on the wave-number $k$ of capillary-wave perturbations. The value of $t^{\prime}$ is real only' between $k=0$ and $k=1 / a$, where $a$ is the radius of the stream of liquid.

Fig. 3. Diagram of conditions governing satellite production. The expression $\lambda / d$ is the - ratio of capillary-wave perturbation wavelength to the diameter of the liquid strean: $l_{b}$, is the lime to break-off of the droplets and is logarithmically related to capillarywave amplitude. The infinity condition is the condition under which satellites do not merge with a primary droplet. Copyright 1977 by International Business Machine "orporation; reprinted with permission.

Fig. 4. Apparatus. The droplet generator consists of the reservoir $(R)$, the throat $(n$ and the orifice $(N)$. The orifice is a small holc cut into a thin stainless steel sheet. The pre-fill tank $(F)$ is a mercury storage tank that facilitates transfer of mercury to the reservoir without breaking the vacuum in the reservoir. The gas-pressure regulator (C) is a Conoflow $H 20 X T$ constant-bleed regulator in series with a standard regulaIor (REG). The PZT piezoelectric crystals $\left(C_{1}, C_{2}\right)$ are powered by the output of an oscillator (Osc; Tektronix $F G 504,40 \mathrm{MHz}$ function generator) which is amplified $\left(A_{1}\right)$ and sent through a 100:1 step-up transformer $(S D)$. The charging electrode $(C E)$ is a cylinder concentric to the stream of liquid, completely surrounding the orifice. The initial driving signal for the charging electrode is supplied by the oscillator $\left(O_{s c}\right)$. This signal is sent through a discriminator $\left(D_{l}\right.$, model 157 dual and/or). into a pre-scaler $(P)$ designed and constructed by Jerry Van Polen of Lawrence Berkeley Laboratory. It is then fed to a pulse generator $(P G$, model $18 \times 1401 P(1)$. amplified $\left(A_{2}\right)$ and fed to the electrode. The amplifier is powered $(A P)$ by a Power Designs model 2005 precision power source. The signal from the discriminator is also fed througin an $A N D(A)$ and a gate $(G)$ into the strobe $(L)$. The $A N D$ and gate are contained in a Tektronix yype $\$ 85$ oscilloscope. The microscope $(M /)$ consists of a Tiyoga 0.08, $3 X$ objective and a Kompens $25 X$ eyepiece. The deflecting sphere 
$(D S)$ is made of silver-plated brass. The collection wells $\left(S F_{1}, S F_{2}\right)$ are constructed of stainless-steel sheet. The liquid collected in the welis is directed out of the apparatus into a storage tank ( $P D$ ). The high voltage supply $(H h$ for the deflecting electrodes $(D E)$ is a standard current-iimited $8 k V M W P C$ power suppiy. A ten-foot section of 3-inch $I D P V C$ pipe $(H)$ houses the deflecting electrodes. Observation windows in the side of the pipe allow visual inspection of the droplets. The deflected droplets are analyzed through the camera viewing port ( $h$ ). The chargecoupled device $(C C D)$ is a Fairchild LineScan camera, model $C C D$ 1410, donalted courtesy of Howard Murphy of Fairchild Semiconductor. It is equipped with at Canon $T V-1650 \mathrm{~mm} / \mathrm{f} / \mathrm{l} .4$ lens. The laser is a Spectra-Physics model 143-01 $4 m W$ He-Ne laser. The multichannel analyzer ( $M A$ ) is a Davidson model $1056 \mathrm{~A}$ multichannel analyzer. The mercury disposal system (S) collects and disposes of the mercury without breaking the vacuum in the housing. The pumping system consists of the mechanical pump ( $A: P)$, the mercury trap ( $M$ ) and the cold trap in a $L N_{2}$ bath $(C T)$, one lobe of which is filled with Zeolite (Z.). Two pressurc valves $(P V)$ relieve high pressures in the apparalus.

Fig. 5. Mode of vibration of the pieznelectric crystals.

Fig. 6. Exploded view of droplet-generator throat.

Fig. 7 . Cienmetry of deflecting electrodes. 
$-12=$

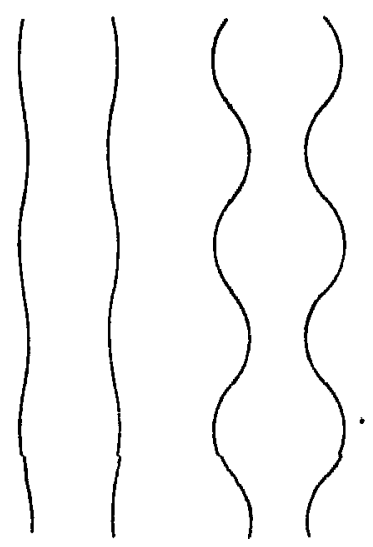

(a)

(b)

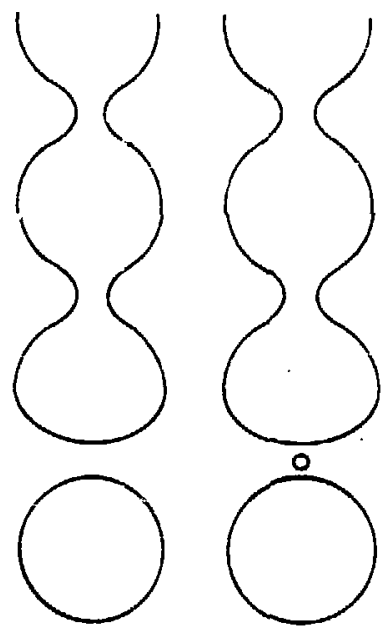

(c)

(d)

$X B L \quad 802-325$

Fioure 1 


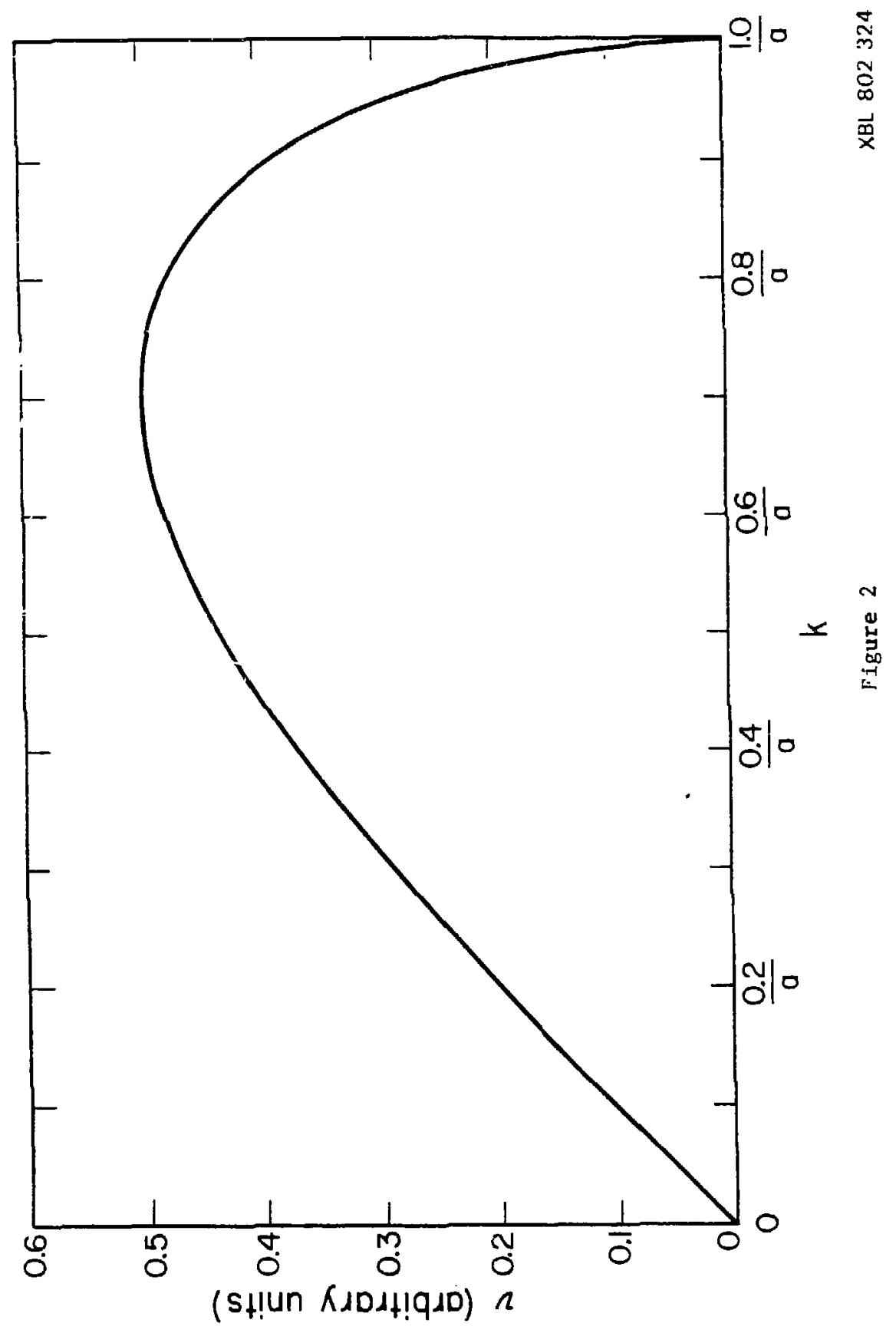




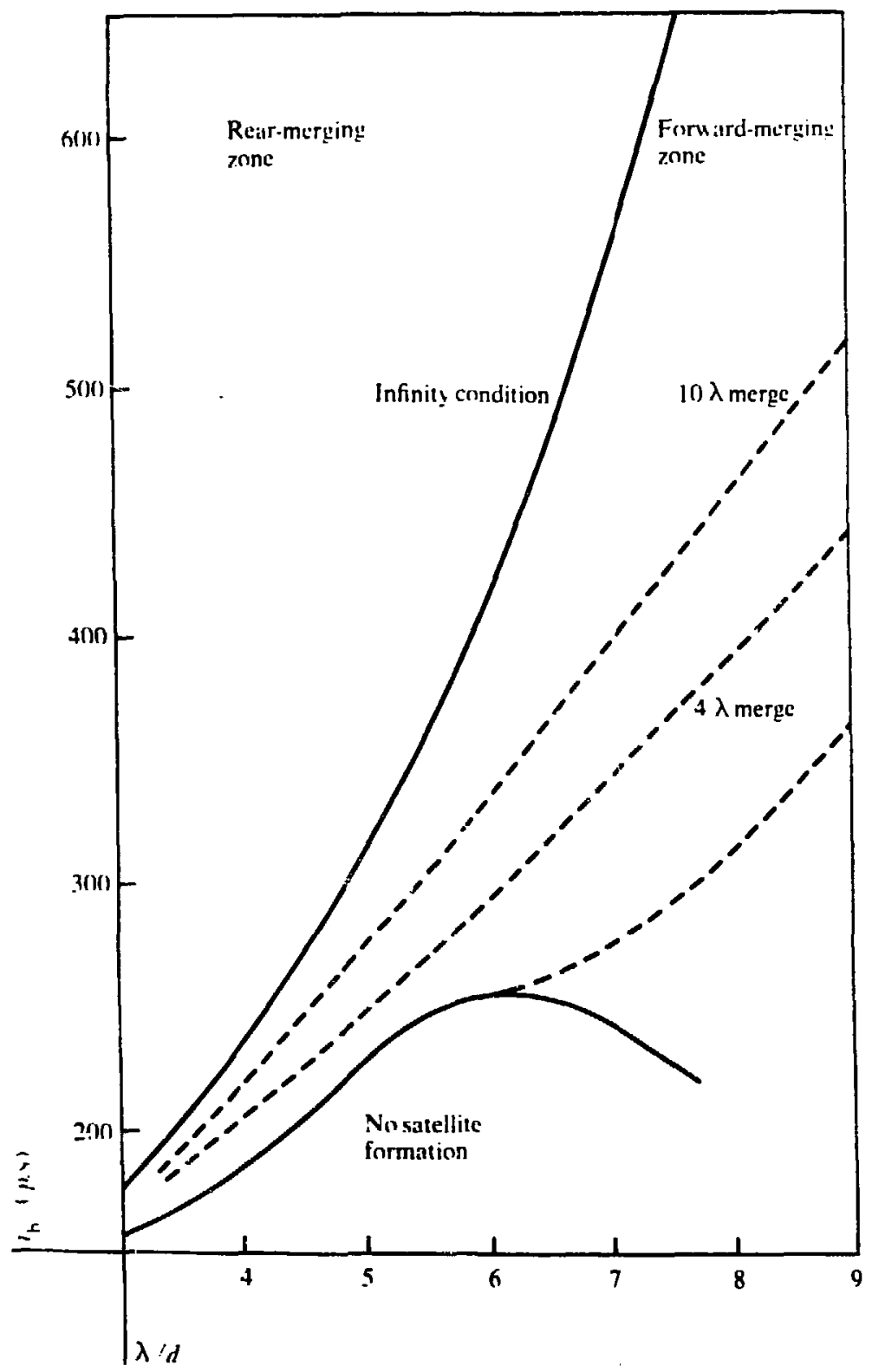

Figure 3

XBL 803-8625 


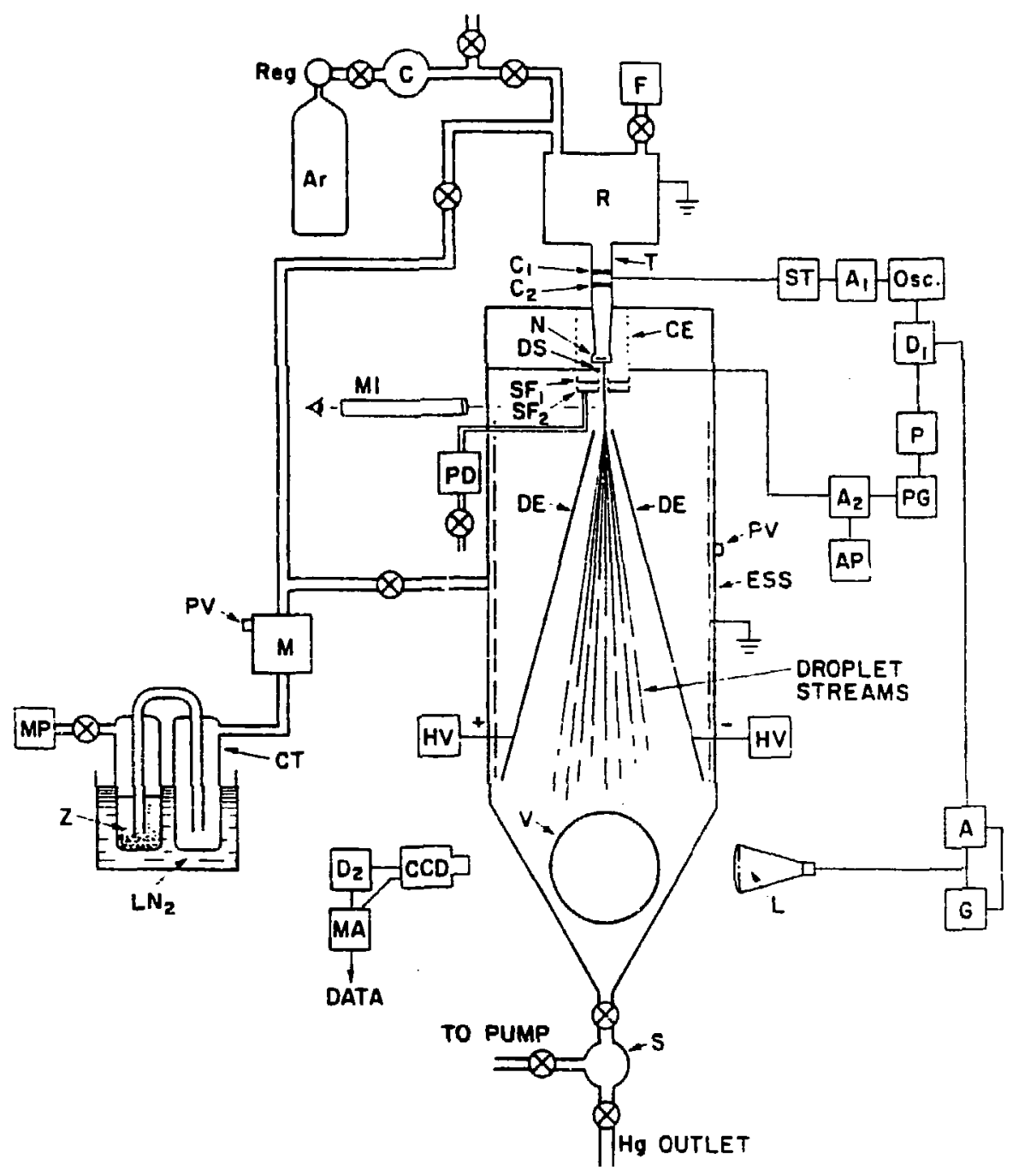

Figure 4

XBL $802 \quad 329$ 


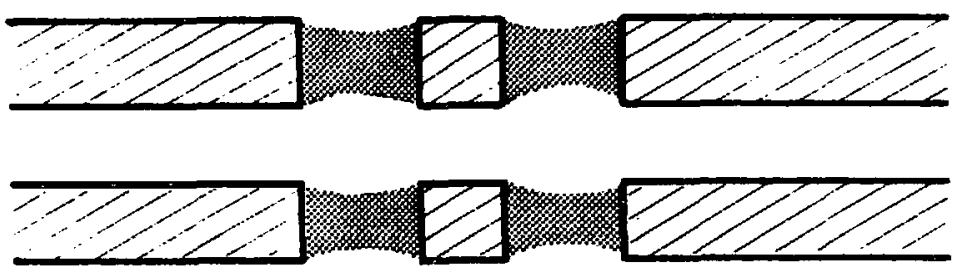

0

n

జ

$\infty$

$\frac{\vec{m}}{x}$
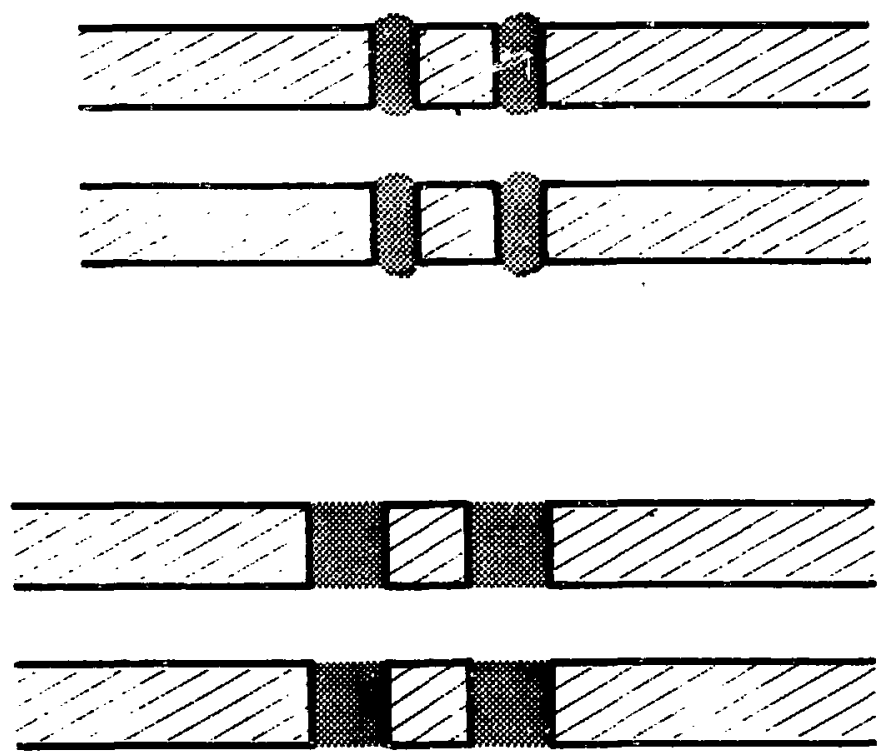

Figur 95 


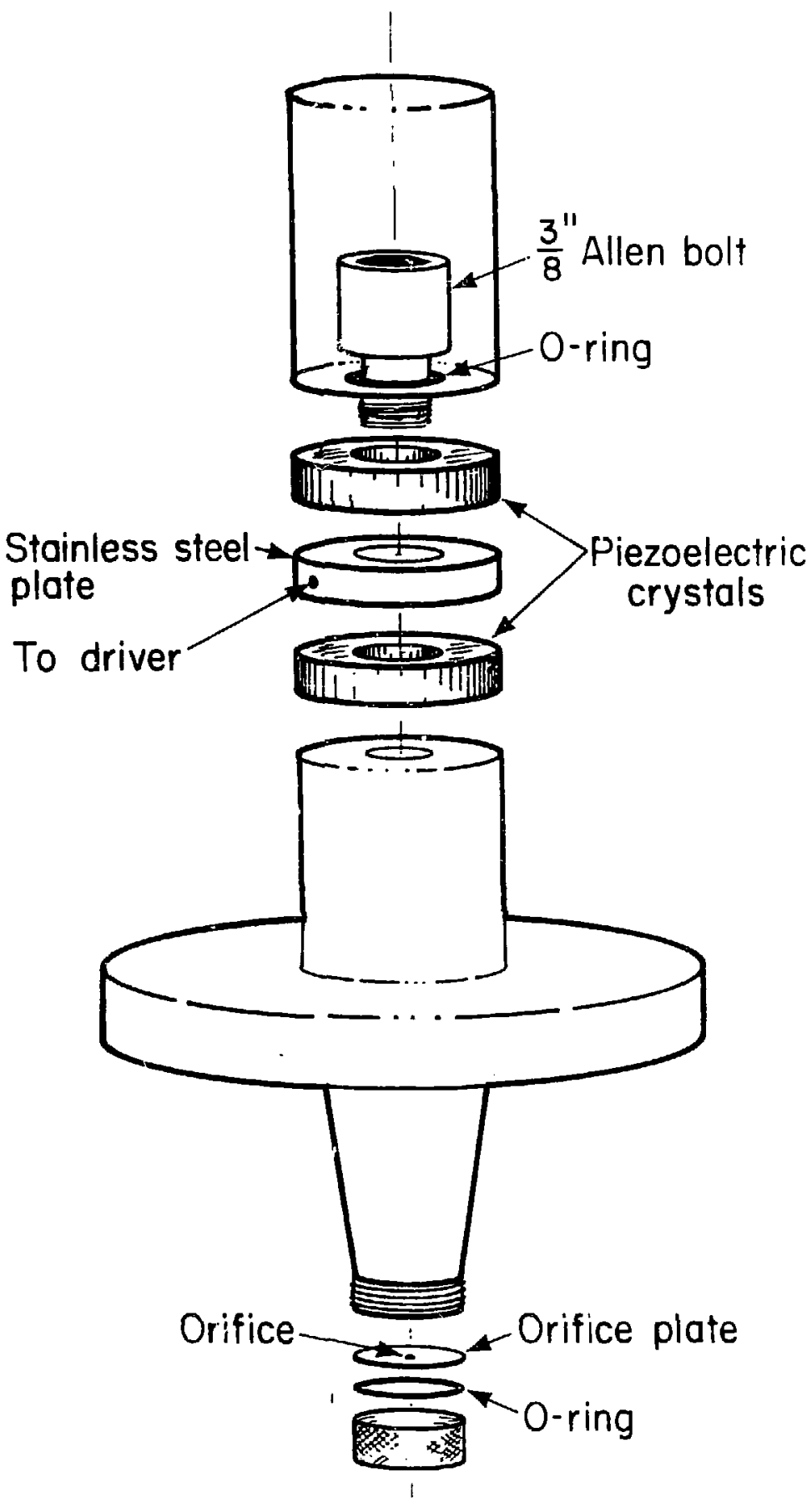




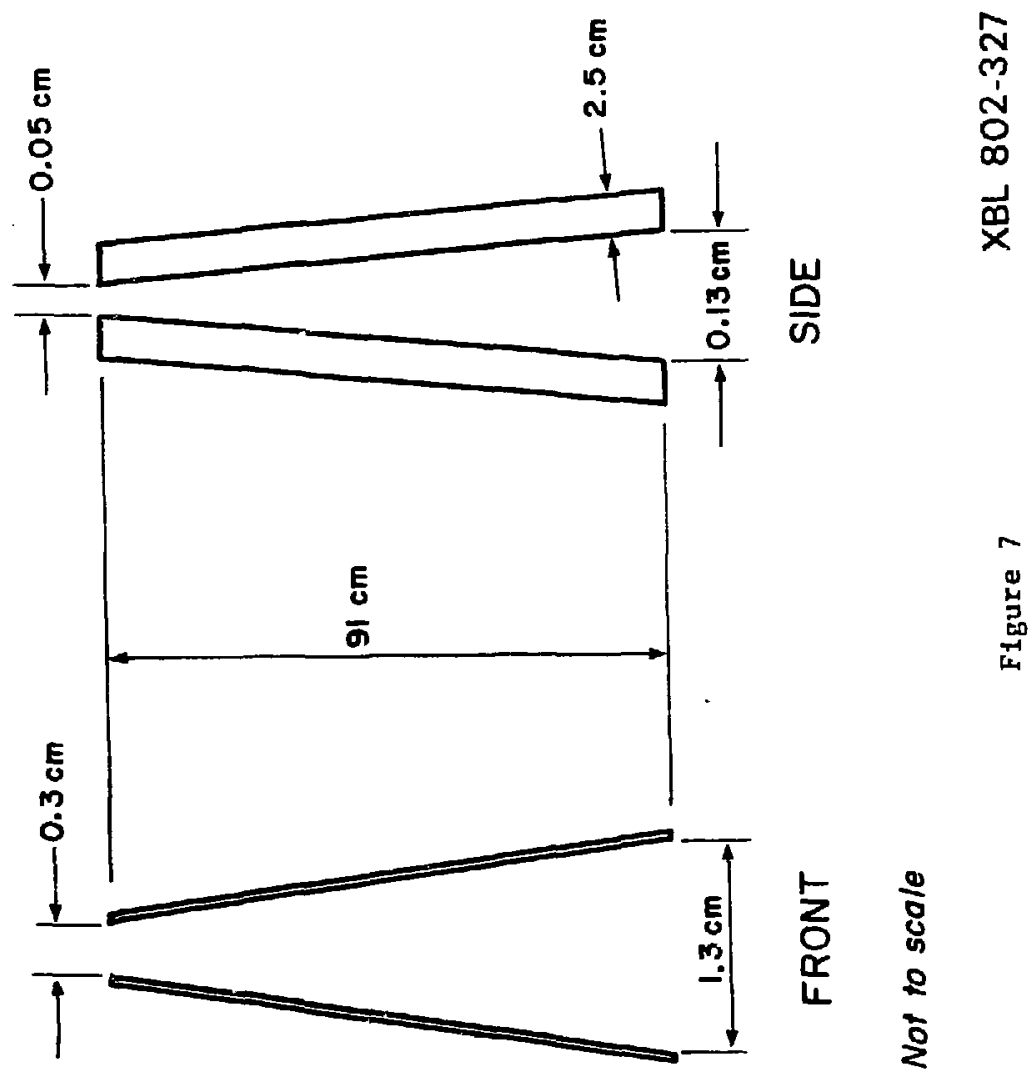

